\title{
Miranda
}

Revue pluridisciplinaire du monde anglophone /

Multidisciplinary peer-reviewed journal on the English-

speaking world

$17 \mid 2018$

Paysages et héritages de David Bowie

\section{Hybridations et effacement du chanteur : l'emploi des codes de la lyric video dans le clip de « Where are we now?»}

\section{Robin Cauche}

\section{OpenEdition}

\section{Journals}

Édition électronique

URL : http://journals.openedition.org/miranda/13241

DOI : 10.4000/miranda.13241

ISSN : 2108-6559

\section{Éditeur}

Université Toulouse - Jean Jaurès

\section{Référence électronique}

Robin Cauche, « Hybridations et effacement du chanteur : l'emploi des codes de la lyric video dans le clip de « Where are we now? » », Miranda [En ligne], 17 | 2018, mis en ligne le 20 septembre 2018, consulté le 16 février 2021. URL : http://journals.openedition.org/miranda/13241 ; DOI : https:// doi.org/10.4000/miranda. 13241

Ce document a été généré automatiquement le 16 février 2021.

\section{cc)}

Miranda is licensed under a Creative Commons Attribution-NonCommercial-NoDerivatives 4.0 International License. 


\title{
Hybridations et effacement $\mathrm{du}$ chanteur : l'emploi des codes de la lyric video dans le clip de "Where are we now?»
}

\author{
Robin Cauche
}

\section{Introduction}

1 Le point de départ de cette étude est une intrusion. Le 8 janvier 2013, par surprise, est publié sur YouTube le clip de la chanson « Where Are We Now? ${ }^{1}$ de David Bowie. ${ }^{2}$ La vidéo est réalisée par le plasticien américain Tony Oursler et annonce la sortie prochaine d'un nouvel album de Bowie, The Next Day. Le chanteur, au fil d'une longue carrière, a créé et incarné un grand nombre d'avatars de lui-même - Ziggy Stardust, Aladdin Sane, The Thin White Duke - notamment présentés et mis en scène dans des vidéoclips - le Pierrot d'Ashes to Ashes, le visage peint de Blue Jean etc. ${ }^{3}$ Ici, c'est sous la forme étrange d'une marionnette au visage vidéoprojeté que l'artiste s'invitait de nouveau dans l'actualité.

2 C'est donc en quelque sorte une intrusion formelle que provoquait Bowie. Pour relayer cette information avec une image récente, journaux et autres chaînes d'information ne disposaient que du clip, soit l'image d'un Bowie hybride, déformé, peu à son avantage. L'œuvre stimulante du vidéaste Tony Oursler entrait alors - un peu par la force, mais cette facétie a quelque chose de réjouissant - au sein même du flux vidéo formaté de l'information télévisée. ${ }^{4}$ Car si à l'ère de YouTube, les clips, mêmes les plus novateurs, sont toujours diffusés à la télévision, ils le sont généralement à des horaires dédiés voire sur des chaînes spécifiquement musicales, en tout cas pas sur les chaînes d'information en continu. En outre, au sein de formes médiatiques très textuelles titres, fil d'actualité, habillages d'antenne etc., l'écrit est omniprésent dans l'information télévisée ${ }^{5}$ - s'ajoutait aussi l'intrusion d'un texte de chanson qui s'affiche 
à l'écran. Notamment ce titre qui, malicieusement, répondait aux questions soulevées par ce retour-surprise de Bowie sous la forme d'une autre question - donc sans répondre vraiment : «Where are we now? » (« Où sommes-nous maintenant? »).

D'emblée, l'étude de ce clip semble cristalliser un grand nombre des questions qui traversent l'étude plus globale de l'œuvre de Bowie : mise en scène de soi (" esthétique de l'invention de soi ", va jusqu'à formuler Shelton Waldrep'), usages et mutations du clip, étrangeté et hybridation - tant physique que générique (dont Matthieu Thibault rend compte par l'oxymore du syntagme avant-garde pop). Sur ces bases, je montrerai comment Bowie et Oursler mettent l'hybridation générique du clip de "Where are we now?» au service d'un effacement du chanteur. Il s'agira d'observer en particulier comment le recours aux codes du genre de la lyric video tend à effacer l'artiste au profit du texte. Dans cette chaîne d'effets se révèle un recours à l'hybridation du chanteur luimême, ainsi qu'à de multiples figures de dédoublement et d'équivoques, au niveau du texte comme de l'image, et en écho aux dédoublements dont la carrière de Bowie est émaillée.

Deux éléments exogènes mettent en effet sur la piste d'une place particulière du texte dans l'effacement du chanteur. Le premier est la pochette palimpseste de l'album The Next Day, qui reprend celle de Heroes. L'ancien titre est rayé de noir, un carré blanc cache partiellement le célèbre portrait en noir et blanc de Bowie, permettant d'écrire en noir le titre du nouvel album. Le second élément est le témoignage d'Oursler luimême, notamment, pour le champ francophone, une interview au Figaro en date du 10 janvier 2013 dont on peut souligner une remarque : «David avait une idée très précise du clip qui devait prendre tout le monde de court. Il voulait une "lyric video" ». (Duponchelle)

\section{Hybridation générique}

5 Le vidéoclip est souvent décrit comme une forme génériquement accueillante. Pour reprendre les termes de Laurent Jullier et Julien Péquignot, le clip «n'invente pas grand-chose » sur le plan des «novations stylistiques » (Jullier \& Péquignot 9), en ce qu'il est un creuset esthétique propice aux références et aux réemplois. D'une part, il permet aux réalisateurs/trices de réinvestir en les concentrant des formes et des motifs canoniques, issus non seulement du long métrage de fiction, mais aussi de la télévision, du concert filmé etc. ${ }^{7}$ D'autre part, il fonctionne souvent comme un «laboratoire visuel » (Gaudin 2015), transposant dans une forme massivement diffusée certaines propositions de l'art contemporain, et « dans certaines occurrences, le clip en arrive à se concevoir comme relevant davantage du domaine des "arts plastiques", au même titre que les installations ou l'art vidéo " (Gaudin 2013, 173). ${ }^{8}$ Toutefois, c'est l'assemblage générique proposé par Bowie et Oursler qui semble ici, non seulement original, mais particulièrement signifiant.

\section{La lyric video}

6 La première forme convoquée par Where are we now ? est la lyric video, et d'après Oursler, c'est Bowie qui souhaitait adopter cette forme, la mentionnant en ces termes. La lyric video est un clip qui illustre une chanson en affichant ses paroles écrites. Cette forme prend racine dans les pratiques amateures de partage des paroles de chanson sur 
internet, dans une tendance globale du clip à jouer avec les mots écrits ${ }^{9}$, ainsi que dans les modalités d'écriture des chansons en vue d'une interprétation en chœur (le karaoké et ses équivalents historiques). Autour de 2010, les maisons de disques s'approprient cette formule illustrative pour créer des lyric videos officielles. Diffusées sur internet ${ }^{10}$, elles servent à promouvoir la diffusion des chansons, souvent dans l'attente d'un autre clip officiel plus « traditionnel » - plus long à produire et plus coûteux.

7 Dans l'émergence de cette forme nouvelle se joue en premier lieu un double mouvement d'interprétation: au sens de performance, afficher le texte permet de faire apprendre la chanson, et donc de la diffuser - de faire chanter, si l'on veut - plus facilement; au sens d'herméneutique, afficher le texte lui accorde une importance accrue en tant que système de signes poétiques, qui s'offre donc frontalement à l'analyse. En outre, cette place dominante du texte dans la promotion des chansons, en particulier dans le domaine de la pop, va plutôt à l'encontre des clichés selon lesquels ces chansons, ciselées pour leurs sonorités, ne méritent pas qu'on s'y attarde textuellement. L'essor récent des lyric videos semble donc le signe et le vecteur de changements dans la diffusion des chansons, qui s'appuie ainsi sur une place renforcée du texte dans la promotion de l'objet-chanson. ${ }^{11}$

Généralement, le corollaire de cette place nouvelle du texte est un retrait provisoire de l'artiste : absent.es ou discret.es dans les lyric videos, les interprètes reprennent leur place à l'écran dans un autre vidéoclip, diffusé ensuite et produit avec plus de moyens économiques et techniques. ${ }^{12}$ Or, chez Bowie, il n'y a pas deux clips successifs, mais bien un seul, qui emprunte la formule textuelle des lyric videos sans affirmer par ailleurs cette spécificité. À cet égard, le titre donné à la vidéo sur YouTube ne mentionne pas qu'il s'agit d'une lyric video - là où fréquemment, un clip textuel sera repéré par la formule «lyric video » ou un équivalent ajoutée au titre de la chanson. Ainsi, Bowie choisit la lyric video pour son principe textuel, sans se conformer aux nouveaux usages de la promotion des chansons sous l'effet desquels cette forme est apparue. Puisqu'il en élude les enjeux commerciaux (la promotion soutenue d'une chanson diffusée successivement sous deux modes officiels d'illustration en vidéo), on peut penser que ce sont les effets d'interprétation et d'appréhension du texte, en somme les implications pragmatiques d'un affichage systématique du texte de chanson, qui intéressent l'artiste : et parmi eux, un retrait relatif de l'interprète.

\section{Art vidéo et installation}

9 Le deuxième champ esthétique convoqué par ce clip est celui de l'art contemporain. Comme le rappelle Antoine Gaudin, la forme du vidéoclip peut parfois relever du domaine de l'art contemporain, en tant qu'elle est un laboratoire propre à l'expérimentation. Le cas de Where are we now? est un peu différent, puisque Tony Oursler importe sa pratique préalable de plasticien et vidéaste au sein de la forme génériquement accueillante du vidéoclip. Il y met ainsi en scène une installation vidéo créée pour l'occasion, mais caractéristique de son style. En effet, il met souvent en scène des corps médiatiques morcelés, ainsi que le décrit Michael Rush :

[S]es «marionnettes", faute d'un mot plus adéquat, [...] sont des personnages filiformes dont la tête est remplacée par un coussin ovale sur lequel Oursler projette la vraie tête parlante d'un acteur [...] (Rush 118) 


\section{de concerts en 1997, interroge la représentation du corps de chanteur. Pour Bowie,} choisir de se couler dans le moule de cette poupée vidéo, c'est ainsi charger d'emblée la mise en images de «Where are we now? » de l'imaginaire que ce dispositif véhicule, et que Paul Ardenne décrit sous la formule de « corps incommunicant » (Ardenne 11). Son aspect grossièrement factice (visage animé sur un corps-peluche immobile) est un premier signe de recul dans la représentation de soi : un corps partiel est projeté dans une installation vidéo, qui est elle-même filmée pour un clip vidéo. Mais les marionnettes étranges d'Oursler paraissent aussi particulièrement propres à véhiculer l'angoisse et la souffrance. ${ }^{13}$ Bowie, ainsi mis en scène pour interpréter la chanson, endosse alors le rôle de "bonimenteur pathétique " que révèle encore Ardenne (Ardenne 11); le pathos du dispositif et la mélancolie de la chanson entrent en résonnance pour informer d'emblée toute lecture de la chanson - à l'invitation, on l'a $\mathrm{vu}$, de ce texte qui s'écrit.

Plus spécifiquement, intégrer le visage de Bowie au dispositif d'Oursler implique deux éléments plutôt rares dans la mise en scène des chanteurs dans les clips, ainsi que l'entrevoit Oursler lui-même : "On a construit un cadre très spécial pour cadrer et capter son visage qui est une icône en soi » (Duponchelle). D'une part, le corps est condamné à l'immobilité, ceci à deux niveaux : celui de Bowie au tournage enserré dans un dispositif de captation, celui de la marionnette du clip dans un dispositif de vidéoprojection. D'autre part, le chanteur n'est plus qu'un visage: métonymique, évocateur, mythique, visage-icône certes, mais néanmoins signe d'un corps morcelé, caché, disparaissant. Le corps de Bowie, en même temps qu'il est proposé à l'audiospectateur/trice sous une forme surprenante, lui est donc nettement refusé sous sa forme la plus attendue (selon la formule canonique du chanteur de clip, si l'on veut) : bien souvent un corps entier magnifié par la mise en scène, énergétique et volontiers dansant.

De la lyric video à l'installation, Where are we now? convoque donc un spectre très large des usages et pratiques de la vidéo. La plasticité du clip permet ce rapprochement de deux formes qui semblent éloignées, mais qui questionnent en fait, chacune à leur manière, la place du chanteur dans la mise en image de sa chanson: dans les deux cas sous le mode de l'effacement, soit au profit d'un texte, soit en une représentation morcelée, hybride et médiatisée.

\section{Deux motifs}

13 Si ce n'est ces deux pôles esthétiques, le clip s'ancre également dans la référence à deux massifs esthétiques à trouver du côté de la peinture. Des massifs qui, s'ils ne constituent sans doute pas des genres, sont en tout cas des motifs récurrents et canoniques.

Le premier est celui de l'atelier d'artiste, ici espace ouvert dans l'esprit loft, qui laisse se déployer un foisonnement d'objets divers et entrevoir discrètement les rouages du dispositif technique. Ainsi, la vidéo qui sert de fond à la marionnette est projetée sur un écran blanc dont on peut voir le support au sol; on distingue également des câbles électriques, des sources de lumière, un ordinateur allumé etc. Ce cadre de l'atelier d'artiste fait évidemment signe vers l'envers de la création artistique, mais surtout vers la construction d'une mise en scène de soi, entre personnage de fiction, persona et éthos de créateur. 
Le second motif est celui de la vanité, et sa composition de symboles voués à figurer le passage du temps et la duplicité d'une vie teintée de mort. Dans ce clip, des symboles forts et plus ou moins classiques sont convoqués : cristal taillé, plaque de verre, cadre vide, reflets, chaîne de métal, moulages, flocon de neige, mannequin, entre autres exemples. En outre, certains détails proposent une réactivation de la vanité à l'aune de l'installation vidéo. On reconnaît notamment au fond de la pièce des rangées de cassettes DVCAM (dans leurs caractéristiques boîtiers violets), comme un signe remis au goût du jour du passage du temps, qui résonne particulièrement avec la construction successive des avatars de Bowie dans divers clips.

Considérer ces deux motifs permet de prendre en charge le caractère symbolique de ces éléments, sans pour autant chercher à faire de Where are we now? un puzzle, une énigme desquels un sens univoque et caché serait à décrypter (une lecture quasi anagogique, eu égard à la mort prochaine de Bowie). Au contraire, les objets symboliques de la vanité et l'appareillage ostensible de l'atelier d'artiste poussent à lire le clip comme un système construit de signes équivoques qui se montre en tant que tel. En outre, cette alliance de références met l'audio-spectateur/trice de Where Are We Now? sur plusieurs pistes d'interprétation qui se n'excluent pas et qui permettent d'appréhender la profusion des signes, volontiers sur un mode réflexif (lecture biographique, mise en scène de soi, voire métadiscours de Bowie sur une vie émaillée de ces mises en scènes de soi), mais pas nécessairement (la vanité met sur la piste d'une lecture nostalgique de la chanson qui n'est pas forcément biographique). Il convient donc de prendre en compte le caractère équivoque du dense réseau de signes proposés par Bowie et Oursler, au sein duquel l'errance du spectateur est relativement guidée par ces quelques pistes.

\section{Sous le signe du dédoublement : monstruosité et équivocité}

Sur le socle de cette hybridation générique initiale, les procédés de dédoublement sont nombreux dans le clip. Les motifs de la vanité et de l'atelier d'artiste mettent d'abord sur la piste de la duplicité (plusieurs statuts, plusieurs pistes d'interprétation pour chaque signe) ; qui plus est, certains éléments du clip sont dédoublés, et apparaissent plusieurs fois avec des statuts différents: les paroles de la chanson sont à la fois chantées et écrites, et David Bowie lui-même est montré sous plusieurs apparences.

\section{Hybridation du chanteur}

En premier lieu, Bowie est figuré, on l'a vu, sous la forme d'une sorte de marionnette à laquelle il ne prête que son visage. Qui plus est, cette marionnette est bicéphale, la seconde tête étant celle d'une femme à l'expression neutre et immuable. Cette hybridité se lit alors à plusieurs niveaux. Elle rappelle l'androgynie de certains avatars de Bowie - en écho à une liberté sexuelle largement assumée -, et formule l'hybridité monstrueuse du corps comme un niveau supérieur du refus de se représenter comme chanteur de clip selon les codes attendus. En terme énonciatif, cette figure hybride est un coup de force, puisqu'elle fait du pronom "we ", qui est sujet syntaxique du refrain, à la fois un singulier (le " nous » d'un « je-Bowie ", qui prend dans les couplets la forme d'un «I» au singulier, mais que cette apparence hybride affirme comme multiple en 
soi) et un pluriel (le «nous » de l'adresse fictive à une personne inconnue, une femme si on veut suivre l'indice donné par ce second visage, qui met la lecture nostalgique des paroles sous les auspices de la chanson d'amour perdu ; un «nous » qui implique aussi l'auditeur/trice dans cette lecture-errance du clip).

En outre, le ventre noir de la marionnette devient à plusieurs reprises une surface de vidéoprojection pour certains mots de la chanson. Se joue alors un double mouvement ontologique. Une marionnette immobile, inanimée, prend vie - d'une certaine manière - par la projection d'un visage; mais dans le même temps, elle est régulièrement rappelée à son caractère d'objet en devenant écran. Construction et effacement, apparition et disparition, vie et mort, là encore, c'est une pensée de la mise en scène de soi-chanteur dans la duplicité qui transparaît.

\section{Dédoublement du chanteur}

S'il est représenté comme une créature hybride au statut problématique, Bowie est également dédoublé par le montage. En effet, au début de la troisième minute du clip, un mouvement de caméra fait sortir de l'écran de projection pour montrer le "vrai " David Bowie, humain, sans le truchement de la projection, et en pied. Puis a lieu un raccord-regard, paradoxal refus d'un champ/contrechamp entre Bowie et sa marionnette, qui se trouve alors filmée de dos. Un Bowie créateur, mis en scène tenant en main des papiers griffonnés (brouillon de la chanson? croquis de l'installation? du clip?) regarde son installation depuis l'arrière, le tout au sein d'une esthétique de l'atelier d'artiste - mentionnée plus haut - qui appuie ce statut de créateur. Il prend donc une distance sur l'installation qui sert de support au clip, et en outre, il l'observe depuis un point de vue qui occulte son propre visage vidéoprojeté dans le dispositif.

21 Ce dédoublement assorti d'un recul est d'ailleurs renforcé par le caractère nécessairement médiatique de ce raccord-regard. En effet, le visage de la marionnette et ce Bowie en pied ne peuvent cohabiter dans l'espace-temps du clip que par le recours à l'enregistrement vidéo puis au montage. Dans les deux cas : par une médiatisation de sa propre image. De plus, le statut de ce second Bowie est lui-même particulièrement équivoque, à la fois spectateur et démiurge de cette mise en scène de soi.

\section{Un déploiement du texte}

Mais au-delà de ces deux niveaux de dédoublement du chanteur, l'équivocité est aussi essentielle au niveau de la chanson, c'est-à-dire de ce texte qui s'affiche à l'écran comme un appel à l'interprétation.

Sur le plan thématique d'abord, "Where are we now? » est une chanson au ton nostalgique qui articule deux temporalités et deux espaces: un passé berlinois qui résonne avec la "période berlinoise" de Bowie ${ }^{14}$, et un présent d'énonciation nettement embrayé ("we », "now ») mais très flou. Cette structure est mise en valeur par la stratification visuelle du clip. L'installation joue sur la disposition d'espaces et d'écrans dans la profondeur du champ, et le montage ajoute une strate textuelle plane par-dessus la profondeur de cette image filmée.

En termes énonciatifs, ce texte tend à rapprocher les trois entités mises en valeur par Stéphane Hirschi parmi les outils de la cantologie : homme privé, chanteur et canteur. Ainsi le clip semble prendre en charge cette mise en crise formelle de l'incarnation du 
texte, dont l'énonciateur est tout à la fois Bowie autobiographe, Bowie conteur et Bowie vecteur d'une entité énonciatrice purement fictive, qui existe dans le texte avant d'être incarnée.

En termes stylistiques, le texte joue lui aussi sur les équivoques et les sens multiples. Quelques exemples simples révèlent l'ouverture interprétative de ce texte: la référentialité complexe des déictiques «now » et «we » (voir supra) ainsi que la forme récurrente de la question (donnant son titre à la chanson) ouvrent le sens, de même que des jeux de mots basés sur la polysémie ("walking the dead », dont la traduction en français peine à épuiser la richesse sémantique ${ }^{15}$ ) ou sur la syntaxe (" you know you know you know» se lit à la fois comme une répétition et comme une suite de propositions enchâssées). En outre, la chanson contient de nombreux mots allemands, certains peuvent être familiers, d'autres restent énigmatiques. Paradoxalement, l'affichage du texte vient à la fois élucider ces mots en langue étrangère et déployer leurs virtualités poétiques et sonores - il s'agit principalement de noms de lieux, qui conservent donc leur caractère évocatoire.

Par ailleurs, l'affichage du texte de la chanson qui caractérise ce clip incite certes à l'interprétation, mais semble encore la diriger. La typographie, qui fait signe vers la casse des machines à écrire et mime les bavures de l'encre, informe l'écoute du texte vers une lecture tournée vers le passé: nostalgique voire autobiographique. L'apparition erratique et imprévisible des mots à l'écran figure et accentue le caractère flottant d'une recherche de sens. Enfin l'écriture des paroles met aussi en valeur d'autres événements textuels du clip, en premier lieu ses textes à valeur de générique : « david bowie » au début, et « a film by tony oursler » à la fin du clip, qui renforcent son caractère de construction, formellement signé de part et d'autre par ses deux créateurs. Qui plus est, on lit également dans le clip les mots « $\mathrm{m} / \mathrm{s}$ Song of Norway ", sur le Tshirt de Bowie. Ils évoquent directement l'errance, le voyage ("Norway», la Norvège, mais aussi le « $\mathrm{m} / \mathrm{s}$ » pour motorship qui indique que « Song of Norway » est le nom d'un navire) et la chanson ( «song »), tout en faisant précisément référence à Hermione Farthingdale, compagne et muse de Bowie, qui le quitte à la fin des années 1960 pour tourner dans la comédie musicale Song of Norway ${ }^{16}$. Ainsi, on peut lire ce texte selon un axe aussi bien thématique qu'autobiographique, et là encore, si la mise en images guide l'interprétation de la chanson, c'est en conservant toutefois son équivocité.

L'attitude de recul de Bowie dans ce clip est donc multiple. D'une part, il met en crise sa propre représentation, hybride, voire regardant sa création; d'autre part, grâce à l'affichage des paroles de la chanson, il propose de se confronter au texte avant tout pour ses virtualités poétiques et littéraires, amorçant des pistes interprétatives sans oblitérer toutefois son ouverture herméneutique.

\section{La mise en scène d'un effacement progressif}

\section{Apparition et disparition}

Plus qu'un recul, on peut observer dans Where are we now? une posture d'effacement de la part de Bowie. Celui-ci semble amorcer un mouvement de disparition de son image, de son corps, et ce au profit du texte de la chanson: en somme un effacement du chanteur au profit de l'œuvre. 

jouant sur le refus de cette apparition. Un travelling porté avance dans un couloir sombre, approche de la marionnette et donc du visage de Bowie, provoquant ainsi un effet d'attente et de dévoilement de ce dispositif surprenant. De plus, un jeu de cadrage participe à cacher le plus longtemps possible le visage dans le hors-champ interne, derrière les barreaux d'un escabeau; ceci est renforcé par les deux premiers textes écrits (le titre de la chanson et le nom de David Bowie), qui eux aussi viennent barrer l'image et la cachent donc partiellement. À la fin de ce plan, la chanson commence, mais c'est alors le play-back qui est refusé. Le visage de Bowie reste bouche close sur la première phrase: "Had to take the train from Potzdamer Platz", qui commence d'ailleurs par l'ellipse du pronom, comme première ouverture interprétative. Ce n'est qu'après un plan de coupe d'archive en noir et blanc que le play-back commence, par la phrase "You never knew that ». Non seulement en apparaissant à l'écran, Bowie refuse d'emblée le statut de chanteur dans le dispositif formaliste du play-back, mais c'est donc par le pronom " you » qu'il entame l'interprétation du texte, invective en forme d'invitation (à interpréter, selon les deux acceptions du terme mentionnées plus haut: chanter la chanson, et se saisir du texte poétique).

De même, le tout dernier plan du clip tend à confirmer cette figuration d'un effacement. Après les dernières phrases de la chanson, Bowie sort du dispositif qui contraint son visage à l'immobilité, et disparaît en trois temps. Son visage, face caméra et yeux regardant l'objectif, s'éloigne d'abord, surcadré par un écran qui a sa forme. Une fois sorti du cadre, c'est son ombre qui passe sur le mur blanc désormais affiché sur le visage de la marionnette, signe de la présence évanescente, fuyante, projetée, du chanteur encore présent à l'état de trace. Pour finir, l'image d'une figure à deux têtes vides, creusées, clôt ce clip qui met ainsi en scène la sortie du chanteur.

Cet effacement qui achève le clip semble un geste particulier dans la vidéographie de Bowie, où celui-ci joue davantage à apparaître qu'à disparaître. Il est par exemple omniprésent dans les vidéos tournées par Mick Rock pour «Life on Mars » et par Stanley Dorfman pour « Heroes » en 1973, cadré selon plusieurs échelles, et à l'image dans l'intégralité des plans. Le fond blanc et l'abondance de très gros plans, notamment du visage (déjà !) dans Life on Mars font d'ailleurs signe vers une esthétique du shooting photo, et quoi qu'il en soit à un processus de présentation, d'apparition, d'incarnation de la chanson. En quelque sorte, c'est Bowie qui y est la chanson puisqu'elle ne peut se dérouler en son absence. À titre de comparaison également, la fin du clip de «Where are we now? » peut être formellement rapprochée de celle de «Blue Jean ${ }^{17}$, où Bowie se dédoublait déjà par le montage afin d'assister, spectateur, à l'interprétation de sa chanson, par lui-même, dans un cabaret. Une fois la chanson terminée, le Bowieinterprète s'éloigne lentement à reculons vers le fond de la scène pour en sortir, tandis que la caméra effectue un travelling arrière. Toutefois, au contraire de Where are we now ?, le clip ne laisse pas le temps à Bowie de sortir du cadre de la scène par le fond, car une foule de spectateurs/trices de dos envahit l'image et cache la star par le premier plan. La mise en perspective de ces deux scènes finales met en lumière leur différence de traitement. Dans Blue Jean, Bowie assiste à sa propre sortie de scène en majesté, chassé du cadre par la foule de ses fans; dans Where are we now?, il semble plutôt orchestrer avec recul et modestie son propre retrait, et prend cette fois le temps de sortir du cadre par lui-même, laissant son ombre planer, ses mots résonner, et figurant son absence en un visage comme déserté. 


\section{Passer derrière le texte}

Ainsi, la présence de Bowie dans le clip est mise en scène sous le mode de l'effacement, du refus, de la suspension de présence, voire de la disparition. Cela est sensible dans l'installation, dans des jeux de cadrage, mais est également figuré par cet envahissement des mots sur l'écran propre à la lyric video.

Lors des différents refrains apparaît à plusieurs reprises l'image de la marionnette pardessus laquelle s'écrivent les mots "Where are we now? ». Ainsi mise en forme, cette question aux accents métaphysiques résonne aussi au premier degré. "Où sommesnous? » demandent les deux têtes de la marionnette : derrière le texte! David Bowie, en un jeu de cache-cache figural, semble affirmer qu'en tant que chanteur, il est désormais en deçà de ses textes. La référence aux miroirs, projecteurs, écrans, prismes, ombres et reflets qui peuplent ce clip-vanité se charge alors d'un sens nouveau, puisqu'on peut ici voir la lyric video comme un dispositif en miroir. La chanson, mise en voix dans l'espace profond et stratifié du studio d'artiste, se traduit en mots via la planéité d'un écran pour être projetée vers les spectateurs/trices, symétriques du chanteur, invité.es à s'emparer de la chanson pour l'interpréter. Si Bowie joue à disparaître, c'est bien pour projeter avec force l'interprétation de la chanson vers l'audio-spectateur/trice du clip.

\section{Conclusion}

À différentes échelles, il semble donc que le clip imaginé par Bowie et Oursler, réalisé par ce dernier, amorce un effacement de l'image du chanteur au profit du texte. Au niveau structurel, l'hybridation générique mariant lyric video, installation vidéo, atelier d'artiste et vanité est particulièrement propice à un discours réflexif sur la place du chanteur, la mise en scène de soi, et cet escamotage symbolique de l'icône derrière l'œuvre. Au niveau des figures, un chanteur hybride, morcelé et dédoublé cède sa place au texte même de sa chanson, derrière lequel il s'efface littéralement.

De plus, Where are we now? semble un geste fort au sein de la vidéographie du chanteur. Certes, le clip met en scène l'effacement de l'artiste grâce à son hybridité générique, ses réseaux de sens, ses figures de dédoublement, de duplicité et de disparition. Mais cela est renforcé si l'on considère la carrière de Bowie : ainsi mis en perspective, à la pointe d'une longue série de clips, et dans la logique d'une mise en scène de soi à laquelle ces clips ont participé, Where are we now ? constitue une rupture. Au contraire de clips comme celui de «Blue Jean » ou de «Life on Mars », celui-ci est présenté comme le lieu où l'artiste peut - enfin? - choisir cet effacement, et mettre en scène librement sa propre sortie.

Les lyric videos proposent quasi systématiquement un nouveau régime de présence de la star qui chante: l'interprète en est généralement absent.e, ou présent.e à l'état de signes discrets - en tout cas rarement filmé.e. La lyric video joue alors avec les attentes d'un second clip qui dévoilera la star. Ici, ce sous-genre du clip est réintégré par Bowie au clip lui-même, et c'est bien une seule vidéo, en présence du chanteur - une présence en crise - qui illustre la chanson. Là où la lyric video participe habituellement d'un effet de révélation, l'emploi des codes du clip textuel dans le seul clip, hybride, de "Where are we now? », met ces caractéristiques au service d'un effacement, d'un recul 
du chanteur au profit du texte de la chanson. Mais si l'on considère, là encore, ce clip à l'échelle plus vaste de la carrière de Bowie, celui-ci propose aussi un recul de l'interprète au profit de son œuvre, dont il anticipe la postérité, et qu'il offre ainsi tout entière à l'interprétation.

\section{Now?}

J'ai tâché d'éviter ici une lecture rétrospective et téléologique de la chanson, qui aurait consisté à faire de Where are we now ? un clip déjà crépusculaire, remarquant que Bowie avait su mettre en images, et en particulier en clips, sa propre mort en janvier 2016. Tout au plus, je propose cette nuance : en synchronie, le clip investit la lyric video pour proposer une nouvelle posture de l'artiste face à son œuvre - et en deçà d'elle; en diachronie, ce clip semble préparer la disparition réelle de Bowie. Where are we now? n'est pas - pas encore - un clip sur la mort de Bowie. Mais sa mise en images transforme sans aucun doute cette chanson nostalgique écrite au passé. D'une certaine manière elle la retourne temporellement, pour en faire également, au futur, un questionnement de Bowie sur sa propre pérennité, son « héritage $~^{18}$ officiel.

À ce titre, la chronologie des vidéos publiées aux alentours de la mort de Bowie par sa maison de disques ${ }^{19}$ est particulièrement intéressante. Son premier clip posthume (pour "I Can't Give Everything Away ») est une lyric video, comme si la postérité par le texte, proposée dans Where are we now?, était effectivement mise en œuvre pour illustrer ses chansons, au moment où il est devenu impossible de le filmer, lui. Viennent ensuite un grand nombre de vidéos illustrant des titres de la comédie musicale Lazarus, toujours textuelles, mais affichant un volume de texte largement réduit : quelques mots écrits, et en particulier son très symbolique titre. $\mathrm{Si}$ «Lazarus » fait référence à celui qui, dans la Bible, est ressuscité, le personnage principal de la comédie musicale est quant à lui un extra-terrestre ayant forme humaine: dans les deux cas, des êtres marqués là encore par la duplicité de leur statut. Enfin, bien après ces vidéos au volume textuel décroissant, paraît en octobre 2016 un live de Michael C. Hall, interprète principal de la comédie musicale, chantant «Lazarus ». Au sens propre, Bowie se trouve alors officiellement réincarné. Ainsi par exemple, c'est Hall qui parut à la cérémonie des Brit Awards 2017, pour accepter en son nom la récompense posthume d'artiste masculin de l'année («British Male Solo Artist»). Il paraît assez clair que Lazarus (la comédie musicale, mais aussi la chanson éponyme et son clip réalisé par Johan Renck) met en scène la disparition du chanteur ${ }^{20}$. Mais d'une manière plus diffuse, au moment où disparaît Bowie se révèle également un passage graduel par des formes textuelles. Ceci participe d'une part à une réincarnation progressive - quoique transitoire - dans Lazarus, et d'autre part à une dissolution de l'artiste dans son œuvre même. Et cette transition par un effacement du chanteur au profit du texte semble, si ce n'est programmée, tout au moins amorcée, avec le clip de « Where are we now? ». 


\section{BIBLIOGRAPHIE}

Ardenne, Paul. « Corps incommunicants ». in Tony Oursler, catalogue de l'exposition « Tony Oursler : dispositifs ». Paris : Flammarion/Éditions du Jeu de paume, 2005, 11-17.

Carpenter, Alexander. « "Give a man a mask and he'll tell the truth": Arnold Schoenberg, David Bowie, and the Mask of Pierrot. » Revue Intersections n 302. Toronto, Canada, 2010: 5-24.

Daney, Serge. «Que demande le clip ?» (Libération, 2 octobre 1985). in Cinéjournal vol. 2, 1983-1986. Paris : Cahiers du cinéma, 1998, 229-231.

Daney, Serge. « Mondino l'as de la hanche » (Libération, 30 octobre 1987). in, Le Salaire du zappeur. Paris, P.O.L, 1993, 136,140.

Delaune, Benoît. « Une application à la chanson enregistrée du modèle transtextuel : transphonographie, intraphonographie, autophonographie et leur application dans la "trilogie berlinoise" de David Bowie ». in Chanson et intertextualité, Ed. Céline Cecchetto, Presses Universitaires de Bordeaux, 2011. 49-63.

Duponchelle, Valérie. « David Bowie : les secrets du clip surprise de Tony Oursler ». lefigaro.fr (en ligne), 10 janvier 2013, consulté le 17/03/2015.

http://www.lefigaro.fr/musique/2013/01/10/03006-20130110ARTFIG00447-david-bowie-lessecrets-du- clip-surprise-de-tony-oursler.php

Fontaine Rousseau, Alexandre. « Testaments filmés. David Bowie (1947-2016). ». Revue 24 images n 176, Montréal.Canada, 2016. 36-37.

Gaudin, Antoine. « Le vidéoclip. De la forme brève cinématographique au médium autonome ». in Sylvie Périneau (dir.) Les formes brèves audiovisuellesUniversité de Limoges, 2013, 169-185.

Gaudin, Antoine. « Le vidéoclip, laboratoire expérimental des industries culturelles : innovations technologiques et expériences esthétiques à l'ère numérique ». in Expanded Cinema, revue Écrans n ${ }^{\circ}$. Paris : Garnier, 2015. 163-177.

Hirschi, Stéphane. Chanson : l'art de fixer l'air du temps. Paris : Les Belles Lettres, 2008.

Jullier, Laurent et Julien Péquignot. Le clip, histoire et esthétique. Paris : Armand Colin, 2013.

Montal, Fabrice. « Le clip dans la chaîne des images : tout est consommé ». Revue 24 images n 48, 1990. 48-50.

Parfait, Françoise, Vidéo : un art contemporain. Paris : Editions du regard, 2001.

Rush, Michael. L'art vidéo. Londres : Thames \& Hudson, 2003.

Thibault, Matthieu. David Bowie : l'avant-garde pop. Marseille : Le Mot et le reste, 2013.

Waldrep, Shelton. The æsthetics of self-invention: Oscar Wilde to David Bowie. Minneapolis: University of Minnesota Press, 2004, 232 p.

\section{NOTES}

1. Afin de distinguer dans le texte les chansons et leurs clips qui, de fait, portent le même titre, j'emploierai les guillemets pour noter les chansons, et les italiques pour noter les clips. 
2. Pour des raisons éditoriales, cet article ne contient - malheureusement - pas d'image extraite du clip étudié. La consultation du clip Where are we now ? est néanmoins aisée, sur la chaîne Youtube officielle de David Bowie, par exemple. Elle éclairera à coup sûr la lecture du présent article.

3. À ce sujet, voir (Carpenter), qui propose une étude de cas sur le Pierrot lunaire incarné par Bowie dans le clip Ashes to Ashes, et revient sur les autres figures incarnées par Bowie dans une section intitulée « Bowies's masks ».

4. Qu'on me permette ici d'appuyer cette assertion sur ma propre expérience, et ce à double titre. D'abord, en tant que téléspectateur, j'ai le vif souvenir des images de ce clip diffusées dans l'émission Télématin sur France 2, ainsi que sur les chaînes d'information en continu BFMTV et iTélé. En outre, étant alors responsable de postproduction de la chaîne de télévision musicale Melody, j'ai moi-même intégré ce clip à l'Agenda, le magazine d'actualité de la chaîne.

5. À ce titre notamment, la stylistique des chaînes d'information paraît mondialement unifiée.

6. Voir (Waldrep), je traduis « the æsthetics of self-invention ».

7. Serge Daney l'exprimait en une formule choc (sans doute de fait un peu exagérée) : «Un clip ce n'est donc pas, comme on dit, "un petit film”, c'est le faux résumé d'un grand film introuvable. » (Daney 1985, 230). Plus critique, Fabrice Montal résumait quant à lui en 1990 : «L'allusion peut faire référence aux grands genres de l'histoire du cinéma (l'image expressionniste, le film noir, le western, etc.), quand elle ne consiste pas en la copie pure et simple de plans de classiques » (Montal 48).

8. Ce double ancrage du clip, en tension entre formes longues classiques (long métrage de fiction notamment) et formes expérimentales, est par ailleurs révélé en diachronie dans la pensée de Serge Daney. Deux ans après l'article mentionné ci-dessus, celui-ci revient en effet sur son propre jugement pour proposer une lecture des clips moins structurale qu'esthétique : « On a dit et redit, un peu vite, que le clip était comme un résumé de grand film [...] Certes, mais ce ne sont peutêtre pas ces clips là les meilleurs. » (Daney 1987, 136-137).

9. Voir notamment le chapitre «Le clip aux prises avec les mots» (Jullier et Péquignot 114-120).

10. Sur le site de partage de video Youtube, en premier lieu.

11. Le présent article n'étant pas frontalement consacré aux lyric videos en tant que massif formel, il ne paraît pas le lieu d'un développement volumineux sur ce genre pourtant encore peu documenté. À toutes fins utiles, précisons néanmoins que les lyric videos, ancrées dans une série culturelle des chansons écrites, sont l'objet de ma thèse de doctorat en cours - sous la direction de Martin Barnier (Université Lumière Lyon 2) et André Gaudreault (Université de Montréal).

12. Mentionnons ainsi à titre d'exemple la chronologie de deux succès récents illustrés par une lyric video et un clip officiels. Pour « Chained to the Rythm » de Katy Perry, une version « (Lyric Video) » est diffusée sur Youtube le 9 février 2017, puis une version «(Official) » le 21 février 2017. De même pour la chanson "Shape of You» d'Ed Sheeran, illustrée d'abord par une « [Official Lyric Video] » diffusée le 5 janvier 2017, puis par une « [Official Video]» le 30 janvier 2017.

13. «Ce que les hommes ont à adresser aux hommes est souvent chargé d'agressivité, de violence, de demande de réparation ou de compassion (les plaintes et les pantins de Tony Oursler) [...]» (Parfait 228), je souligne.

14. Voir (Delaune)

15. Il faudrait traduire à la fois « promener les morts » comme on promène un chien (« to walk a dog »), «faire marcher les morts », et aussi sans doute " réveiller les morts », puisque " walking the dead » résonne très directement du syntagme «the walking-dead », c'est-à-dire « les mortsvivants ».

16. Andrew L. Stone, 1970. Notons le nom d'un des deux auteurs du livre dont la comédie musicale est une adaptation: Milton Lazarus. Il semble rétrospectivement trouver sa place logique dans le réseau de signes qui annoncent et figurent la fin de carrière de Bowie. 
17. Le clip réalisé par Julien Temple en 1984 est extrait d'un court-métrage d'une vingtaine de minutes intitulé Jazzin' for Blue Jean, il sera récompensé du Grammy Award du meilleur clip en 1985.

18. Je reprends ici l'un des termes fédérateurs qui sous-titrait le colloque dans lequel cette étude prenait initialement place : « New ways ever free : paysages et héritages de David Bowie ».

19. Sur la chaîne Youtube officielle https://www.youtube.com/user/DavidBowieVEVo

20. Au sujet du caractère crépusculaire du clip réalisé par Johan Renck, avec la collaboration de Bowie, pour sa chanson "Lazarus ", voir (Fontaine Rousseau). C'est aussi l'avis du metteur en scène de Lazarus, Ivo van Hove : «Avec Lazarus, il est pour moi tout à fait clair que Bowie a voulu mettre en scène sa propre mort». Rapporté par leparisien.fr (en ligne) : «Lazarus, la comédie musicale de David Bowie, à Londres fin octobre ", 25 juillet 2016, consulté le 10 août 2017. http:// www.leparisien.fr/flash-actualite-culture/lazarus-la-comedie-musicale-de-david-bowie-alondres-fin-octobre-25-07-2016-5993905.php

\section{RÉSUMÉS}

« Where are we now? » : « Où sommes-nous maintenant? » demandait David Bowie en 2013 dans un clip réalisé par le plasticien Tony Oursler. Le chanteur, qui avait su façonner son image, notamment par des vidéoclips, et qui figurerait bientôt sa propre mort dans Lazarus (2016), questionnait donc déjà sa disparition et sa postérité. Le clip hybride notamment deux formes qui semblent éloignées : la lyric video affichant les paroles de la chanson, et l'installation vidéo dans le champ de l'art contemporain. Cet article propose d'approcher ce clip très dense à la lumière de l'équivocité et du dédoublement des signes, ainsi que de l'hybridation, tant des genres que du corps de l'artiste. L'affichage des paroles de la chanson, propre aux lyric videos, paraît à la fois inviter à l'interprétation et guider celle-ci sur certaines pistes. En particulier, cette présence forte du texte semble proposer un mouvement de recul de l'artiste au profit de son œuvre, David Bowie s'effaçant derrière un texte pour finalement figurer sa disparition du clip - qui résonne, $a$ posteriori, de la disparition réelle de l'artiste, quelques années plus tard.

"Where are we now?" was the question David Bowie asked in a music video directed by American plastician Tony Oursler, and released in 2013. Over the years, Bowie created many avatars, notably thanks to music videos, and in 2016, he would even metaphorically depict his own death in Lazarus. In Where are we now?, he highlighted the questions of disappearance and posterity in particular. On a formal level, the video refers to two genres which can seem very opposite: on the one hand, lyric videos, that are official textual music videos, featuring the lyrics of the illustrated song; on the other hand, video installation and contemporary art. This paper proposes to analyse this music video in the light of equivocality, ambiguities and duplications within a dense network of signs. It will also consider the hybridization, of genres and of Bowie's body and selfrepresentation as well. The writing of lyrics on screen, which is specific to lyric video as a genre, seems an invitation to interpretation. Specifically, the importance given to the text leads to notice the gradual fading of Bowie himself, as a singer, behind his own song. In the end of the video, he will indeed totally disappear from the screen - a disappearance that echoes afterwards with Bowie's actual death, a few years later. 
INDEX

Keywords : lyric video, music video, hybridization, song lyrics

Mots-clés : lyric video, clip, hybridation, texte de chanson

\section{AUTEURS}

\section{ROBIN CAUCHE}

Doctorant en études cinématographiques et audiovisuelles Université Lumière Lyon 2/Université de Montréal r.cauche@univ-lyon2.fr 